\title{
Comparison between Rectangular and Triangular Patch Antennas Arrays
}

\author{
Yahya S. H. Khraisat ${ }^{1}$, Melad M. Olaimat ${ }^{2} \&$ Sharief N. Abdel-Razeq ${ }^{1}$ \\ ${ }^{1}$ Department of Electrical and Electronics Engineering, Al-Huson University College, Al-Balqa' Applied \\ University, PO Box 50, Al-Huson 21510, Jordan \\ ${ }^{2}$ Department of Electrical Engineering, Amman Faculty of Engineering, Al-Balqa' Applied University, PO Box \\ 2250, Irbid 21110, Jordan \\ Correspondence: Yahya S. H. Khraisat, Department of Electrical and Electronics Engineering, Al-Huson \\ University College, Al-Balqa' Applied University, PO Box 50, Al-Huson 21510, Jordan. Tel: 962-777-060-985. \\ E-mail: yahya@huson.edu.jo
}

Received: December 20, 2011 Accepted: January 10, 2012 Online Published: May 1, 2012

doi:10.5539/apr.v4n2p75 URL: http://dx.doi.org/10.5539/apr.v4n2p75

\begin{abstract}
In this paper, several designs of micorstip arrays antennas, suitable for wireless communication applications, are presented. This paper demonstrates several shapes of microstrip array antennas, such as rectangular and triangular patch antennas array. Specifically, $4 \times 1,2 \times 1$, and single element of both shapes are designed and simulated by a full wave simulator (IE3d). Moreover, this paper presents a comparison between both rectangular and triangular antenna arrays. Since, the resonance frequency of these antennas is $2.4 \mathrm{GHz}$, these antennas are suitable for ISM band and WLAN.
\end{abstract}

Keywords: microstrip antennas array, rectangular microstrip antennas, triangular microstrip antennas, resonant frequency of patch antennas

\section{Introduction}

Modern wireless communication systems require low profile, lightweight, high gain and simple structure antennas to assure reliability, mobility, and high efficiency (Huque et al, 2011). A microstrip patch antenna is very simple in construction using a conventional microstrip fabrication technique. Microstrip antennas consist of a patch of metallization on a grounded dielectric substrate. They are low profile, lightweight antennas, most suitable for aerospace and mobile applications. Microstrip antennas have matured considerably during the past 35 years, and many of their limitations have been overcome (Garg et al., 2001).

The conducting patch can take any shape, but rectangular and circular configurations are the most commonly used configurations. Among the shapes that attracted much attention lately is the triangular shaped patch antenna. (Olaimat \& Dib, 2011; Olaimat \& Dib, 2011; Kimothi et al., 2008; Nasimuddin et al., 2005; Guha \& Siddiqui, 2004; Olaimat, 2010; Saunders \& Aragon-Zavala, 2007; Bahl \& Bhartia, 1980). This is due to their small size compared with other shapes like the rectangular and circular patch antennas. In this paper, several designs of rectangular patch antennas arrays and triangular patch antennas arrays are presented. Specifically, 4 by 1,2 by 1 , and single element of both shapes are designed. Moreover, these designs are simulated using IE3d (full wave simulator). Based on the simulation results, comparison between both rectangular and triangular patch antennas array is achieved. This paper is divided into five sections: the first section is devoted to give an overview of the microstrip antennas. Second section gives a preface of the important parameters in single element designs, for both rectangular and triangular. Third section discusses the patch antenna design and the necessity of the antenna array. Forth section demonstrates the results of the paper as a whole and a comparison between both shapes (rectangular and triangular) is presented. Finally, a brief conclusion is presented in the fifth section.

\section{Single Element Design Issues}

\subsection{Introduction}

A microstrip antenna element can be used alone or in combination with other like elements as part of an array. In either case, the designer should have a step-by-step element design procedure (Garg et al, 2001). Usually, the overall goal of a design is to achieve specific performance at a stipulated operating frequency. If a microstrip 
antenna configuration can achieve these overall goals, then the first decision is to select a suitable antenna geometry. In this paper, both rectangular and triangular patch antenna arrays are designed and compared, as it will be shown in the subsequent sections.

\subsection{Side Length and Resonant Frequency}

Figure 1 shows the geometry of single element of both rectangular and triangular patch antennas. Based on the cavity model, the resonant frequency of the rectangular patch antenna, of length $\mathrm{L}$, can be calculated using the following formula (Garg et al., 2001):

$$
f_{1,0}=\frac{c}{2 L \sqrt{\epsilon_{r}}}
$$

where $c$ is the speed of light in free space, and $\epsilon_{r}$ is the dielectric constant of the substrate.
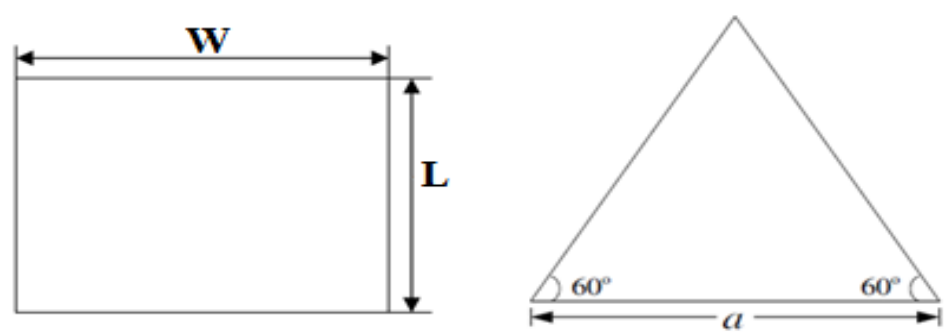

Figure 1. Geometry of both rectangular and equilateral triangular patch antennas

It is worth to mention that, the rectangular patch width $\mathrm{W}$ has a minor effect on the resonant frequency and radiation pattern of the rectangular patch antenna (Garg, et al, 2001).

Regarding the equilateral triangular patch antenna (ETPA), of side length $a$, the resonant frequency can be calculated using the following formula (Garg et al., 2001), (Olaimat \& Dib, 2011), (Olaimat \& Dib, 2011), (Kimothi et al., 2008), (Nasimuddin et al., 2005), (Guha \& Siddiqui, 2004), (Olaimat, 2010), (Saunders \& Aragon-Zavala, 2007), Bahl \& Bhartia, 1980):

$$
f_{1,0}=\frac{2 c}{3 a \sqrt{\epsilon_{r}}}
$$

\subsection{Substrate Selection}

One of major steps in designing a patch antenna is to choose a suitable dielectric substrate of appropriate thickness $\mathrm{h}$ and loss tangent. A thicker substrate, besides being mechanically strong, will increase the radiation power, reduce conductor loss, and improve impedance bandwidth. However, it will also increase the weight, dielectric loss, surface wave loss, and extraneous radiations from the feeder. The substrate dielectric constant $\epsilon_{r}$ plays a role similar to that of substrate thickness. A low value of $\epsilon_{r}$, for the substrate, will increase the fringing field at the patch periphery, and thus, radiated power. Therefore, substrate with $\epsilon_{r}<2.5$ are preferred unless a smaller patch size is desired, and thus we used substrate with $\epsilon_{r}=2.2$ is used in our designs. An increase in the substrate thickness has similar effect on antenna characteristics as the decrease in the value of $\epsilon_{r}$. A high loss tangent increases dielectric loss and therefore reduces antenna efficiency (Garg et al., 2001).

\subsection{Design of Single Element}

Figure 2 shows the design of both rectangular and triangular patch antennas and its dimension. As shown in the Figure, both patches are fed by microstrip line inset feed. A Substrate with $\epsilon_{r}=2.2$ and thickness $\mathrm{h}=1.6 \mathrm{~mm}$ is used in both designs, as well. Figure 3 shows the radiation pattern for both designs in Figure 2. It can be seen that the radiation pattern in both designs is in broadside direction. Both designs are resonating at $2.4 \mathrm{GHz}$ as shown in Figure 4. The reflection coefficient in both designs is falling below $-10 \mathrm{~dB}$. Actually, the reflection coefficient in rectangular is approximately $-40 \mathrm{~dB}$, whereas it is $-30 \mathrm{~dB}$ in triangular. 

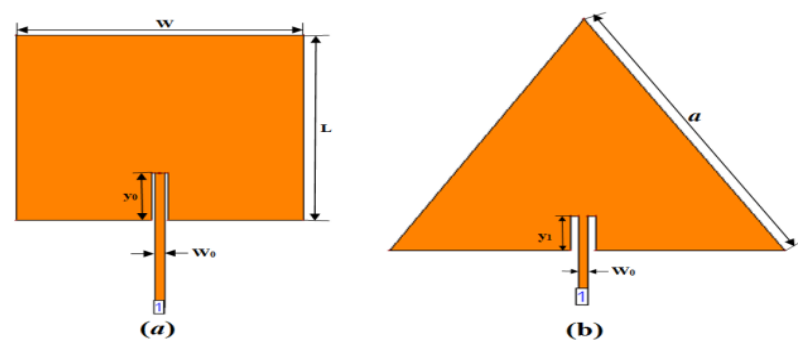

Figure 2. Geometry of patch antenna $a$ ) Rectangular; b) Triangular; with dimensions (in mm): $\mathrm{L}=42, \mathrm{w}=42.4, a=54.9, \mathrm{y}_{0}=13.5, \mathrm{y}_{1}=9$ and $\mathrm{w}_{0}=4.82$

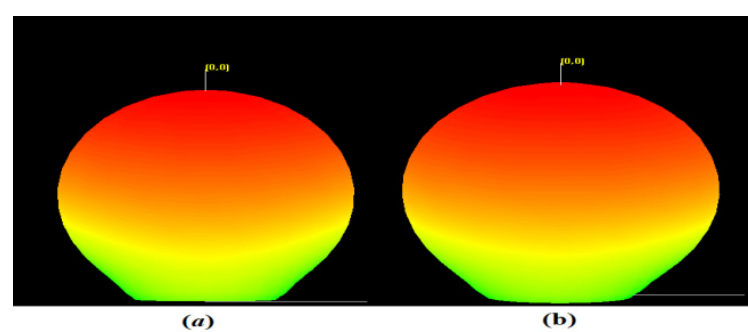

Figure 3. The radiation pattern at 2.4GHz for: $a$ ) Design in figure 2.a; b) Design in figure 2.b

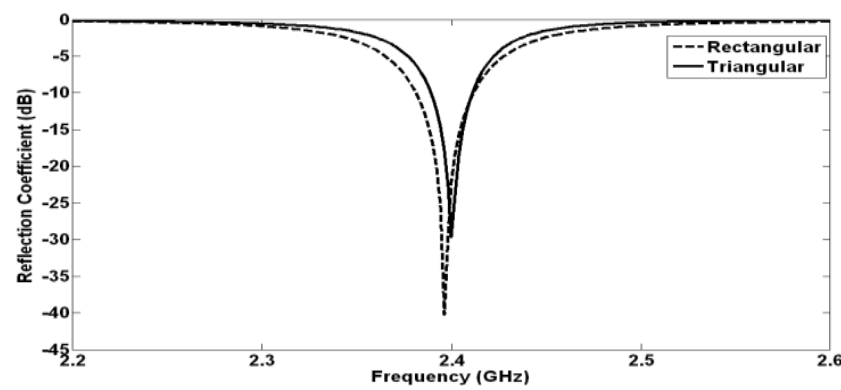

Figure 4. Simulated reflection coefficient (in dB) of the designs shown in Figure 2

\section{Array Design}

\subsection{Introduction}

In certain applications, desired antenna characteristics may be achieved with a single microstrip element. However, as in the case of conventional microwave antenna, characteristics such as high gain, beam scanning, or steering capability are possible only when discrete radiators are combined to form arrays. The elements of an array may be spatially distributed to form a linear, planar, or volume array. A linear array consists of elements located finite distances apart along a straight line. In practice, the array type is usually chosen depending on the intended application (Garg et al, 2001). Feeding methods, that are employed to feed microstrip array in this paper, are parallel and quarter-wave-transformer methods.

\subsection{Design of Linear 2x1 Array}

Actually, in order to make fair comparison, the same substrate used in single element $\left(\epsilon_{r}=2.2\right.$ and thickness $\mathrm{h}=1.6 \mathrm{~mm}$ ), is used in the $2 \times 1$ array. Figure 5 shows the configuration of $2 \times 1$ linear rectangular patch antenna array. To obtain $50 \mathrm{Ohms}$ input impedance, feeding line with width $\mathrm{W}_{1}=4.85 \mathrm{~mm}$ is used. This line is split into two $100 \mathrm{Ohms}$ lines, with width $\mathrm{W}_{\mathrm{o}}=1.41 \mathrm{~mm}$ for each as shown in Figure 5. Figure 6 shows the configuration of $2 \times 1$ linear triangular patch antenna array. The same design procedure, that was used for rectangular, is used in triangular array. It is worth to mention that, the patches (rectangular and triangular) dimensions used in the arrays have same dimensions used in single patches design that are shown in Figure 2. Figure 7 shows the 
reflection coefficient for both designs shown in Figures 5 and 6. Obviously, both designs resonate at approximately $2.4 \mathrm{GHz}$ with different levels of reflection coefficient.

Figure 8 shows the radiation pattern for both designs in Figures 5 and 6 . It can be seen that the radiation patterns in both designs are in broadside direction. But, small side lobes appear in both patterns.

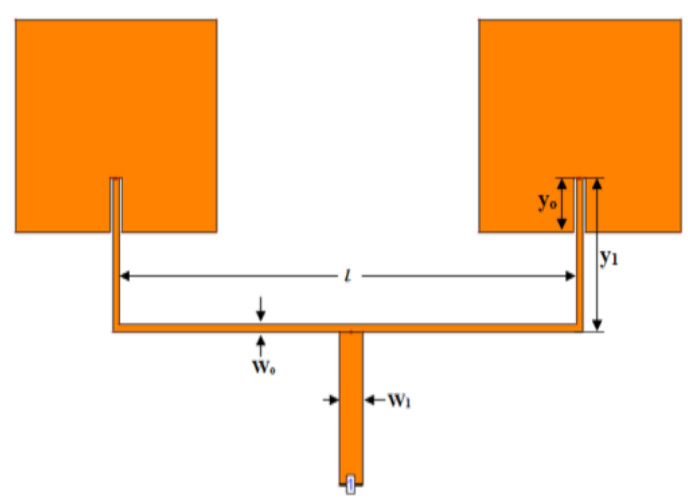

Figure 5. Configuration of $2 \mathrm{x} 1$ linear rectangular patch antenna array; with $\mathrm{W}_{\mathrm{o}}=1.41 \mathrm{~mm}, \mathrm{~W}_{1}=4.89 \mathrm{~mm}, \mathrm{y}_{\mathrm{o}}=10.5$ $\mathrm{mm}, \mathrm{y}_{1}=30 \mathrm{~mm}$, and $l=96 \mathrm{~mm}$

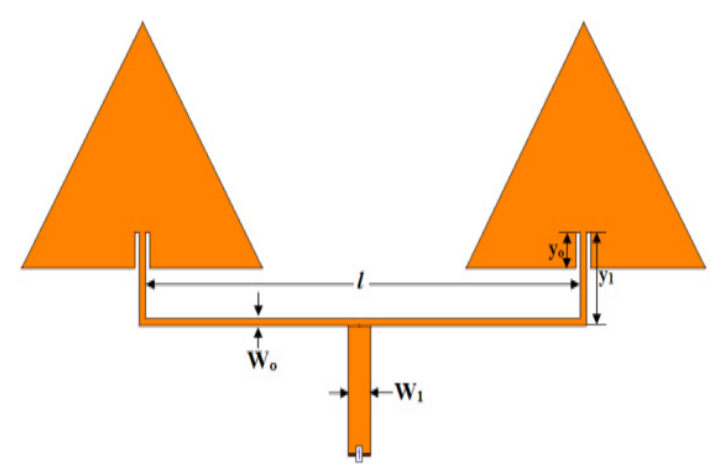

Figure 6. Configuration of $2 \times 1$ linear triangular patch antenna array; with $\mathrm{W}_{\mathrm{o}}=1.41 \mathrm{~mm}, \mathrm{~W}_{1}=4.89 \mathrm{~mm}, \mathrm{y}_{\mathrm{o}}=7 \mathrm{~mm}$, $\mathrm{y}_{1}=18 \mathrm{~mm}$, and $l=96 \mathrm{~mm}$

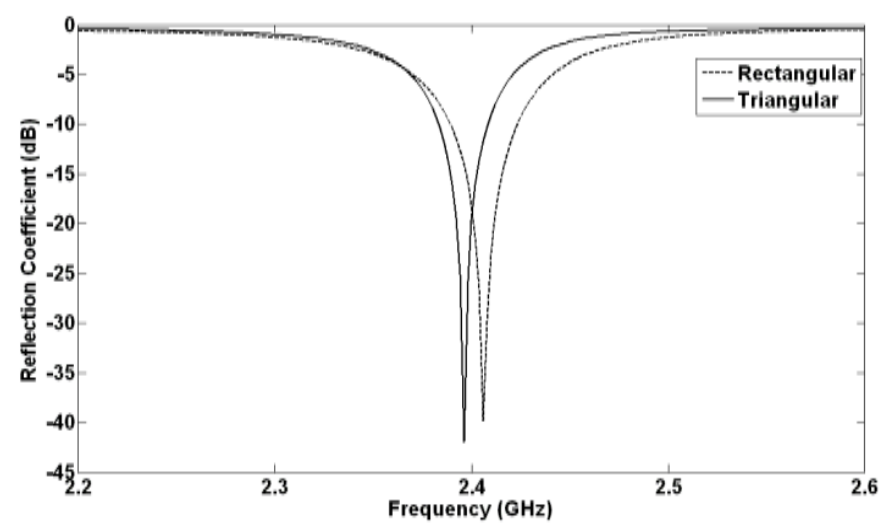

Figure 7. Simulated reflection coefficient (in $\mathrm{dB}$ ) of the designs shown in Figures 5 and 6 


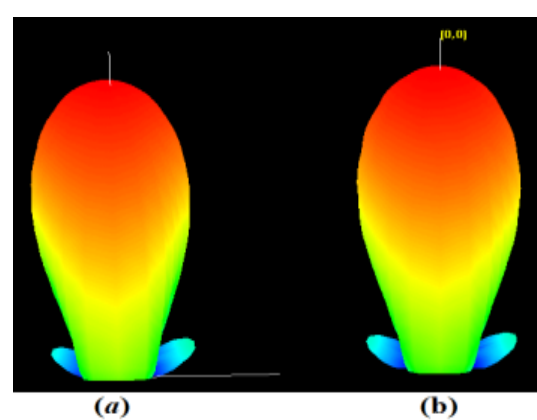

Figure 8. The radiation pattern at $2.4 \mathrm{GHz}$ for: $a$ ) Design in Figure 5; b) Design in Figure 6

\subsection{Design of $4 x 1$ Array}

In an attempt to design $4 \times 1$ array, for both rectangular and triangular, quarter wave transformer is used to feed the elements. Figure 9 shows the configuration of $4 \times 1$ rectangular patch antenna array. All dimensions are shown in the figure. The dimensions of quarter wave transformer are based on calculations. Figure 10 shows the configuration of $4 \times 1$ triangular patch antenna array, as well. In order to compare between both rectangular and triangular 4x1 array, the reflection coefficient of both designs (shown in Figures 9 and 10) are shown in Figure 11. It can be seen both designs resonate approximately at $2.4 \mathrm{GHz}$.

Figure 12 shows the radiation pattern for both designs in Figures 9 and 10. It can be seen that the radiation patterns in both designs are in broadside direction. The side lobes appear clearer in those designs. It is worth to mention that, the side lobe in rectangular patch (Figure 12.a) is worse than that in triangular patch antenna (Figure 12.b) for this array design.

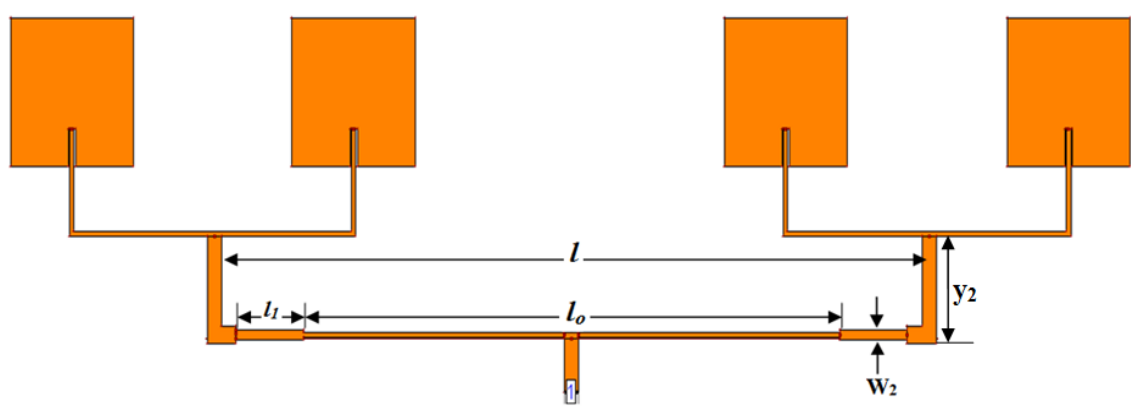

Figure 9. Configuration of $4 \mathrm{x} 1$ rectangular patch antenna array; with $\mathrm{W}_{2}=2.85 \mathrm{~mm}, \mathrm{y}_{2}=30 \mathrm{~mm}, l=201.2 \mathrm{~mm}$, $l_{o}=185 \mathrm{~mm}$ and $l_{l}=23.1 \mathrm{~mm}$

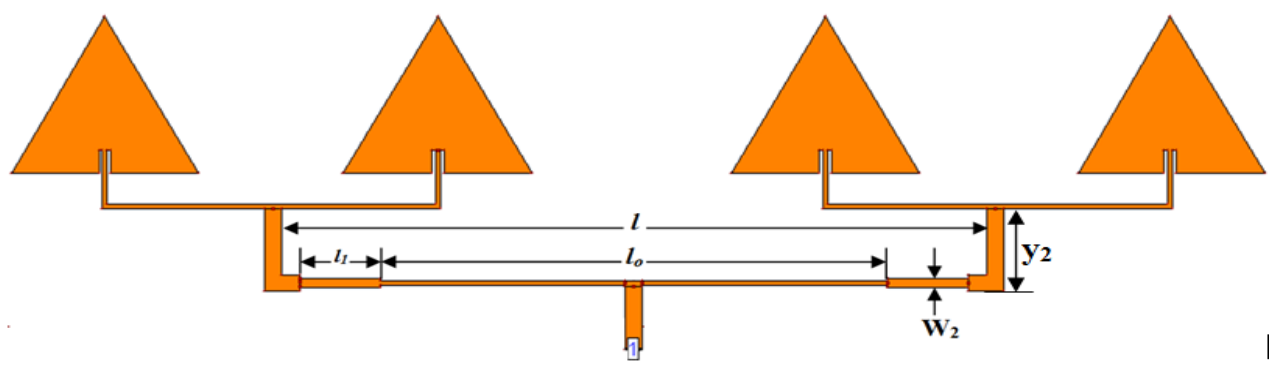

Figure 10. Configuration of $4 \mathrm{x} 1$ triangular patch antenna array; with $\mathrm{W}_{2}=2.85 \mathrm{~mm}, \mathrm{y}_{2}=25 \mathrm{~mm} l=201.2 \mathrm{~mm}$, $l_{o}=185 \mathrm{~mm}$ and $l_{l}=23.1 \mathrm{~mm}$ 


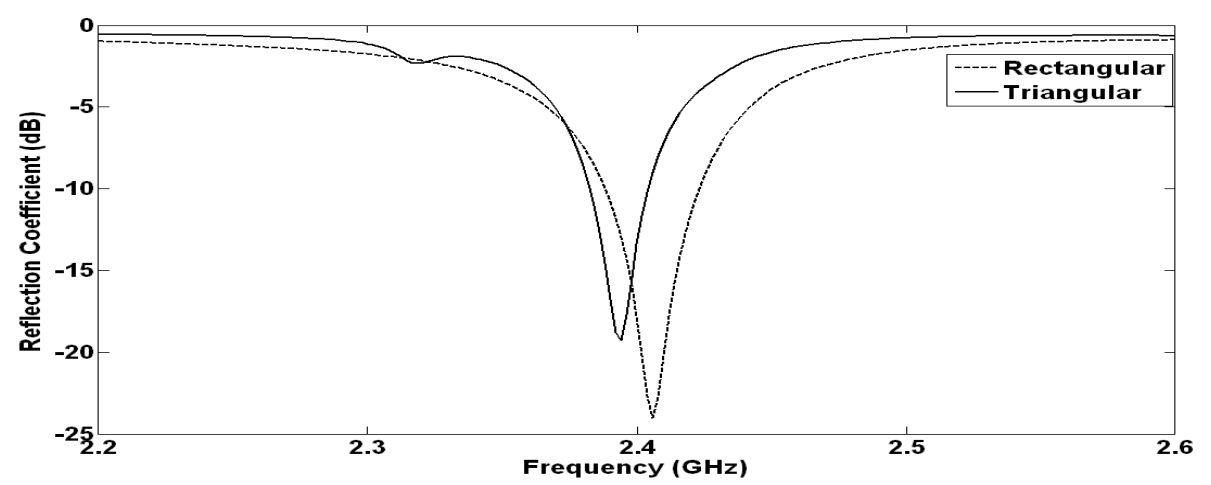

Figure 11. Simulated reflection coefficient (in $\mathrm{dB}$ ) of the designs shown in Figures 7 and 8

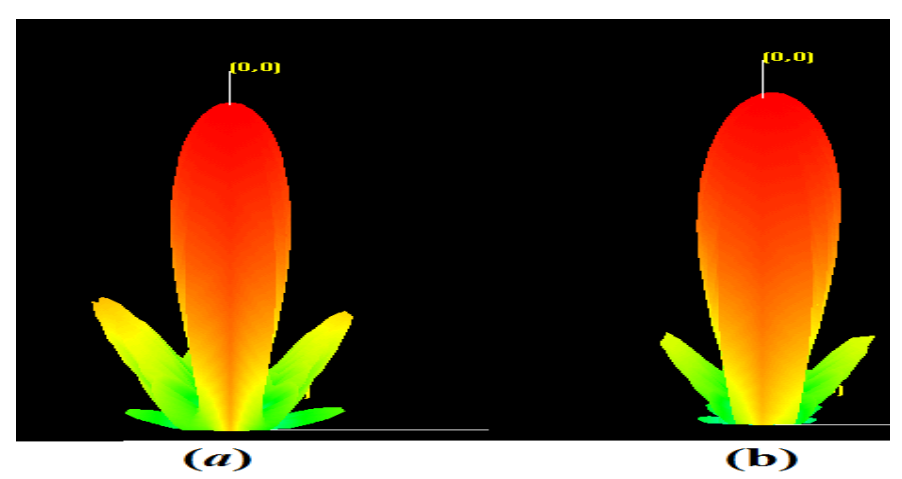

Figure 12. Radiation pattern at $2.4 \mathrm{GHz}$ for: $a$ ) Design in Figure 9; b) Design in Figure 10

\section{Comparision}

Table 1 shows the obtained simulated results. As shown in the table, the results obtained from rectangular patch are very close to those obtained from triangular patch. The reflection coefficient is best in single and $2 \times 1$ array for both shapes (Rectangular and Triangular). It could be because good matching is obtained in those cases. Both gain and directivity, for both shapes (Rectangular and Triangular), are increasing as the number of elements is increased, which is expected. Finally, the side lobe level is increasing as the number of elements is increased as shown in radiation pattern Figures (Figures 3,8 and 12) with the advantage for triangular patch antenna over rectangular patch antenna.

Table 1. Comparison between rectangular (Rect) and triangular (Tri) patch antenna arrays

\begin{tabular}{ccccccc}
\hline Number of Elements & \multicolumn{2}{c}{$\mathrm{S} 11(\mathrm{~dB})$} & \multicolumn{2}{c}{ Gain $(\mathrm{dB})$} & \multicolumn{2}{c}{ Directivity $(\mathrm{dB})$} \\
& Rect & Tri & Rect & Tri & Rect & Tri \\
\hline Single & -40 & -35 & 6.7 & 6.3 & 7.4 & 7.36 \\
$2 \times 1$ & -39 & -41.4 & 9.6 & 9.5 & 10.5 & 10.7 \\
$4 \times 1$ & -23.6 & -19.2 & 12.5 & 12.4 & 13.6 & 13.6 \\
\hline
\end{tabular}

\section{Conclusion}

In this paper, triangular patch antenna arrays were studied, for first time up to our knowledge. Several shapes of both rectangular patch antennas and triangular patch antennas arrays were designed, specifically, $4 \times 1,2 \times 1$ and single element. All designs are compatible for WLAN and ISM application. Good enhancement, on both gain and directivity, is obtained by employing the array techniques. In this paper, we proved the ability of using triangular patch antenna array with same performance of rectangular approximately. Moreover, using triangular patch antenna array, we could obtain better suppression for side lobe level than that obtained using rectangular patch antenna array, especially in $4 \times 1$ array. 


\section{References}

Bahl, I. J., \& Bhartia, P. (1980). Microstrip Antennas. Artech House, Dedham, MA, (Chapter 4).

Garg, R., Bhartia, P., Bahl, I. J., \& Ittipiboon, P. (2001). Microstrip Antenna Design Handbook. Artech House, Boston, London.

Guha, D., \& Siddiqui, J. Y. (2004). Resonant frequency of equilateral triangular microstrip patch antenna with and without air gaps. IEEE Trans. Antennas Propagation, 52, 2174-2177. http://dx.doi.org/10.1109/TAP.2004.832504

Huque, T. I., Hossain, K., Islam, S., \& Chowdhury, A. (2011). Design and Performance Analysis of Microstrip Array Antennas with Optimum Parameters For X-band Applications. International Journal of Advanced Computer Science and Applications, 2, 81-87.

Kimothi, A., Saxena, V., Saini, V. K., \& Bhatnagar, J. S. (2008). Radiations from a Right Triangular Patch Antenna with and without Air Gap. Proceedings of International Conference on Microwave, 154-156.

Nasimuddin, K. Esselle, K., \& Verma, A. K. (2005). Resonant Frequency of an Equilateral Triangular Microstrip Antenna. Microwave and Optical Technology, 47.

Olaimat, M. (2010). Design and analysis of triangular microstrip patch antennas for wireless communication systems. Master Thesis. Jordan University of Science and Technology.

Olaimat, M. M., \& Dib, N. I. (2011). A Study of $15-75^{\circ}-90^{\circ}$ Angles Triangular Patch Antenna. Progress In Electromagnetics Research (PIER), 21, 1-9.

Olaimat, M. M., \& Dib, N. I. (2011). Improved Formulae for the Resonant Frequencies of Triangular Microstrip Patch Antennas. International Journal of Electronics, 98, 407-424. http://dx.doi.org/10.1080/00207217.2010.547811

Saunders, S. R., \& Aragon-Zavala, A. (2007). Antenna and Propagation for Wireless Communication Systems. John Wiley \& Sons, England. 